\title{
Decision-Maker's Risk Preference Based Intuitionistic Fuzzy Multiattribute Decision-Making and Its Application in Robot Enterprises Investment
}

\author{
Liandong Zhou $\mathbb{D}$ and Qifeng Wang $\mathbb{1}$ \\ Logistics and E-Commerce College, Zhejiang Wanli University, Ningbo 315100, China \\ Correspondence should be addressed to Qifeng Wang; lhywqf@163.com
}

Received 29 June 2018; Accepted 4 September 2018; Published 24 September 2018

Guest Editor: Carlos Llopis-Albert

Copyright ( 2018 Liandong Zhou and Qifeng Wang. This is an open access article distributed under the Creative Commons Attribution License, which permits unrestricted use, distribution, and reproduction in any medium, provided the original work is properly cited.

\begin{abstract}
At present, the utilization of hesitation information of intuitionistic fuzzy numbers is insufficient in many methods which were proposed to solve the intuitionistic fuzzy multiple attribute decision-making problems. And also there exist some flaws in the intuitionistic fuzzy weight vector constructions in many research papers. In order to solve these insufficiencies, this paper defined three construction equations of weight vectors based on the risk preferences of decision-makers. Then we developed an intuitionistic fuzzy dependent hybrid weighted operator (IFDHW) and proposed an intuitionistic fuzzy multiattribute decision-making method. Finally, the effectiveness of this method is verified by a robot manufacturing investment example.
\end{abstract}

\section{Introduction}

In 1986, the fuzzy theory of Zadeh [1] was extended to the intuitionistic fuzzy theory by Atanassov [2]. Intuitionistic fuzzy sets contain three parts: membership, nonmembership, and hesitation. With these three parts, intuitionistic fuzzy sets (IFSs) can describe the fuzzy nature world better than the traditional fuzzy sets.

Researchers have made great achievements in the study of intuitionistic fuzzy information aggregation. By using the IFS which are characterized by a membership function and nonmembership functions, $\mathrm{Xu}[3,4]$ developed intuitionistic fuzzy weighted averaging (IFWA) operator, intuitionistic fuzzy ordered weighted averaging (IFOWA) operator, and intuitionistic fuzzy hybrid aggregation (IFHA) operator. S Zeng [5] considered the probabilities and the OWA in the same formulation and proposed the Pythagorean fuzzy probabilistic ordered weighted averaging (PFPOWA) operator. Over the past decades, researchers have developed many operators to solve the multiple attribute group decision-making (MAGDM) problems. Wei [6] proposed induced intuitionistic fuzzy ordered weighted geometric (IIFOWG) operator and induced interval-valued intuitionistic fuzzy ordered weighted geometric (I-IIFOWG) to solve the MAGDM problems. Su et al. [7] extended the induced generalized ordered weighted averaging (IGOWA) operator and developed induced generalized intuitionistic fuzzy ordered weighted averaging (IG-IFOWA) operator. Zeng et al. [8] considered both ordered weighted average operator and induced ordered weighted average and proposed pythagorean fuzzy induced ordered weighted averaging weighted average (PFIOWAWA) operator for MAGDM. Combining intuitionistic fuzzy operators and TOPSIS method together, many multiattribute decision-making (MADM) methods have been developed [9-11]. Based on the TOPSIS method, intuitionistic fuzzy VIKOR methods were introduced $[12,13]$ and the problem of choosing the best alternative due to the incommensurability between attributes had been well solved. Chatterjee et al. [14] integrated the Analytic Hierarchy Process and the VIKOR compromise-ranking method together and constructed a flexible multicriteria decisionmaking (MCDM) framework. Huang et al. [15] extend the VIKOR method to MAGDM with interval neutrosophic numbers (INNs). Meng et al. [16] introduced the prospect theory into MADM with interval-valued intuitionistic fuzzy information. Qin et al. [17] proposed a decision-making 
model by integrating VIKOR method and prospect theory. Xie et al. [18] applied prospect theory and grey relational analysis to stochastic decision-making. Li et al. [19] aggregated the decision-making information in different natural states by using the prospect theory.

Although there are many research achievements on intuitionistic fuzzy information aggregation and methods for solving MADM and MAGDM problems, there are still some drawbacks and research gaps for further research:

(1)Intuitionistic fuzzy numbers (IFNs) contain three parts: membership, nonmembership, and hesitation. Therefore, when using the aggregation operator to rank IFNs, these three aspects should be taken into account simultaneously. But most of the existing aggregation operators are only concerned about two parts: membership and nonmembership. The uncertainty (hesitation degree) of IFNs is often ignored.

(2)In the process of MADM, common aggregation operators often assume that attributes are independent from each other. $\mathrm{Xu}$ [20] proposed a weighting method based on normal distribution. The characteristic of this method is giving a smaller weight to the data that is too high or too low; therefore the effect of larger deviations on integration results can be eliminated as much as possible. However, there is a flaw in this method, the weight is independent of the data which to be integrated and cannot reflect the relationship between data. If the interaction factors of attributes are taken into account in the aggregation operators, decision-makers will be assisted to obtain more accurate decision results.

(3)The existing weight determination methods are mostly focused on subjective weighting [21]. Some objective weight determination methods need to solve the linear or nonlinear programming model $[22,23]$. And computation of these methods is relatively cumbersome and is not suitable for decision-making problems with lots of alternatives and attributes.

(4)Recently, researchers made some progresses in decision-making with risk preferences. Adding risk preferences can affect the decision-maker's psychological factors into the decision-making process. That can reduce the error of decision results and improve the quality of the decision-making. Liu, J. et al. [24] proposed a new model involved risk preferences of decision-makers based on the prospect theory and criteria reduction. Wan et al. [25] developed a new method with interval-valued intuitionistic fuzzy preference relations for solving group decision-making problems. Y. Lin et al. [26] developed a method to determine relative weights of decision-makers depending on preference information. However, the most existing research on risk preferences focuses on priority weights and less researches from the perspective of decision-maker's attitude.

(5)Recently, IFS has been applied to many decisionmaking fields, such as supplier selection $[10,27,28]$ and pattern recognition [29-31]. But there is no application in robot enterprises investment field.

Considering all the problems listed above, this paper focused on the study of intuitionistic fuzzy multiattribute decision-making with decision-makers' different attitudes. And in order to give decision-makers most desirable results, we combined intuitionistic fuzzy theory and risk preference partition theory which is from expected utility theory. In risk preference partition theory, the risk preference attitude of decision-makers can be divided into three categories: risk proneness, risk aversion, and risk neutralness. Then we defined the weight vectors equations according to the attitudes of decision-makers based on the three parts of IFNs. They're objective weights and easy to be calculated. By taking interaction factors of attributes into account, we defined the intuitionistic fuzzy dependent hybrid weighted operator and proposed a decision-making method. The effectiveness of this method is verified by a robot enterprises investment example.

\section{Preliminaries}

Atanassov [1] introduced nonmembership to the Zadeh fuzzy sets (FS)[2] and defined IFS, shown as follows.

Definition 1. $X=\left\{x_{1}, x_{2}, \cdots, x_{n}\right\}$ is an universe of discourse; then

$$
A=\left\{\left\langle x, \mu_{A}(x), v_{A}(x)\right\rangle \mid x \in X\right\}
$$

is an IFS, where for each element $x \in X, \mu_{A}(x): X \longrightarrow$ $[0,1]$ represents the membership, and $\nu_{A}(x): X \rightarrow[0,1]$ represents the nonmembership, with the condition satisfying $0 \leq \mu_{A}(x)+v_{A}(x) \leq 1, \forall x \in X$. And $\pi_{A}(x)=1-\mu_{A}(x)-v_{A}(x)$ is a degree that characterizes the uncertainty or hesitancy of each element $x \in X$ in IFS set $A$. In particular, if $\pi_{A}(x)=0$, $\forall x \in X$, then set $A$ degenerate into Zadeh fuzzy sets. The membership degree, nonmembership degree, and hesitation degree of the IFS effectively extend the representation ability of classical fuzzy sets. For convenience, we can define $\alpha=$ $\left(\mu_{\alpha}, \nu_{\alpha}\right)$ as an IFN, where $\mu_{\alpha} \in[0,1], v_{\alpha} \in[0,1]$, and $\mu_{\alpha}+\nu_{\alpha} \leq 1$. Xu [4] introduced the IFNs operational laws, shown as follows.

Definition 2. $\alpha=\left(\mu_{\alpha}, \nu_{\alpha}\right)$ and $\beta=\left(\mu_{\beta}, \nu_{\beta}\right)$ are two IFNs; then five operational laws are as follows:

$$
\begin{aligned}
\bar{\alpha} & =\left(\nu_{\alpha}, \mu_{\alpha}\right), \\
\alpha \oplus \beta & =\left(\mu_{\alpha}+\mu_{\beta}-\mu_{\alpha} \mu_{\beta}, \nu_{\alpha} \nu_{\beta}\right), \\
\alpha \otimes \beta & =\left(\mu_{\alpha} \mu_{\beta}, \nu_{\alpha}+\nu_{\beta}-v_{\alpha} \nu_{\beta}\right), \\
\lambda \alpha & =\left(1-\left(1-\mu_{\alpha}\right)^{\lambda}, \nu_{\alpha}^{\lambda}\right), \quad \lambda>0, \\
\alpha^{\lambda} & =\left(\mu_{\alpha}^{\lambda}, 1-\left(1-v_{\alpha}\right)^{\lambda}\right), \quad \lambda>0 .
\end{aligned}
$$

Based on operational laws of IFNs above, a weighted averaging operator of IFNs is given by $\mathrm{Xu}$ [4]. 
Definition 3. $\alpha_{i}=\left(\mu_{i}, v_{i}\right)(i=1,2, \cdots, n)$ is a set of IFNs, and letting $\Theta$ be the set of intuitionistic fuzzy numbers, then IFWA : $\Theta^{n} \longrightarrow \Theta$ is defined as follows:

$$
\begin{array}{r}
I F W A_{\omega}\left(\alpha_{1}, \alpha_{2}, \cdots \alpha_{n}\right)=\sum_{i=1}^{n} \omega_{n} \alpha_{n} \\
=\left(1-\prod_{i=1}^{n}\left(1-\mu_{i}\right)^{\omega_{i}}, \prod_{i=1}^{n} v_{i}^{\omega_{i}}\right)
\end{array}
$$

then IFWA is called intuitionistic fuzzy weighted averaging operator, where $\omega=\left(\omega_{1}, \omega_{2}, \cdots, \omega_{n}\right)^{T}$ is the weight vector of $\alpha_{i}(i=1,2, \cdots, n)$ with the condition satisfying $\omega_{i} \in$ $[0,1](i=1,2, \cdots, n), \sum_{i=1}^{n} \omega_{i}=1$. Obviously, by using the IFWA operator to aggregate IFNs, the aggregated value is also an IFN. Thus the loss of information is avoided.

\section{Intuitionistic Fuzzy Dependent Hybrid Weighted Operator}

The IFWA operator only considers the importance of IFNs by using the weight vector, but the risk attitude information inside the IFNs is also very important. In order to exploit the risk attitude information inside the IFNs, we need to introduce similarity degree defined by $\mathrm{Xu}$ [32].

Definition 4. $\alpha_{1}=\left(\mu_{\alpha_{1}}, \nu_{\alpha_{1}}\right)$ and $\alpha_{2}=\left(\mu_{\alpha_{2}}, \nu_{\alpha_{2}}\right)$ are any two IFNs; $\overline{\alpha_{2}}=\left(\nu_{\alpha_{2}}, \mu_{\alpha_{2}}\right)$ is the complement of $\alpha_{2}$; then

$$
S\left(\alpha_{1}, \alpha_{2}\right)= \begin{cases}0.5, & \alpha_{1}=\alpha_{2}=\overline{\alpha_{2}} \\ \frac{d\left(\alpha_{1}, \overline{\alpha_{2}}\right)}{d\left(\alpha_{1}, \overline{\alpha_{2}}\right)+d\left(\alpha_{1}, \alpha_{2}\right)}, & \text { others }\end{cases}
$$

is the similarity degree between $\alpha_{1}$ and $\alpha_{2}$, where

$$
d\left(\alpha_{1}, \alpha_{2}\right)=\frac{1}{2}\left(\left|\mu_{\alpha_{1}}-\mu_{\alpha_{2}}\right|+\left|\nu_{\alpha_{1}}-v_{\alpha_{2}}\right|+\left|\pi_{\alpha_{1}}-\pi_{\alpha_{2}}\right|\right)
$$

is the standard Hamming distance between $\alpha_{1}$ and $\alpha_{2}$.

Definition 5. $\alpha_{i}=\left(\mu_{i}, v_{i}\right)(i=1,2, \cdots, n)$ is a set of IFNs; then the average of the IFNs is defined as

$$
\begin{aligned}
\alpha & =\frac{1}{n}\left(\alpha_{1} \oplus \alpha_{2} \oplus \cdots \oplus \alpha_{n}\right) \\
& =\left(1-\prod_{i=1}^{n}\left(1-\mu_{i}\right)^{1 / n}, \prod_{i=1}^{n} v_{i}^{1 / n}\right)
\end{aligned}
$$

In order to reflect the preferences of decision-makers, we divide the risk attitude information into three kinds: risk proneness, risk aversion, and risk neutralness. Then we redefine the weight equations to extract risk attitude information that inside the IFNs.

Definition 6. $\alpha_{i}=\left(\mu_{i}, v_{i}\right)(i=1,2, \cdots, n)$ is a set of IFNs; then three kinds of risk attitudes are introduced by using different weight equations. (i) Risk proneness weight equation for $\alpha_{i}$ is defined as

$$
\omega_{i}=\frac{\pi_{i}}{\sum_{i=1}^{n} \pi_{i}}
$$

with the condition satisfying $\sum_{i=1}^{n} \omega_{i}=1(i=1,2, \cdots, n)$, $\omega_{i} \in[0,1]$. The degree of hesitancy is $\pi_{i}=1-\mu_{i}-\nu_{i}$. Obviously, the greater the degree of hesitancy, the greater the corresponding weight. Risk proneness decision-makers consider hesitancy as advantage.

(ii) Risk aversion weight equation for $\alpha_{i}$ is defined as

$$
\omega_{i}=\frac{1-\pi_{i}}{\sum_{i=1}^{n}\left(1-\pi_{i}\right)}
$$

with the condition satisfying $\sum_{i=1}^{n} \omega_{i}=1(i=1,2, \cdots, n)$, $\omega_{i} \in[0,1]$. Because of $1-\pi_{i}=\mu_{i}+\nu_{i}$, the greater the degree of hesitancy, the smaller the corresponding weight. Risk aversion decision-makers consider hesitancy as disadvantage.

(iii) Risk neutralness weight equation for $\alpha_{i}$ is based on similarity degree and average of IFNs, defined as follows:

$$
\omega_{i}=\frac{S\left(\alpha_{\sigma(i)}, \alpha\right)}{\sum_{i=1}^{n} S\left(\alpha_{\sigma(i)}, \alpha\right)}, \quad i=1,2, \cdots, n
$$

with the condition satisfying $\sum_{i=1}^{n} \omega_{i}=1(i=1,2, \cdots, n)$, $\omega_{i} \in[0,1]$, where $S\left(\alpha_{\sigma(i)}, \alpha\right)$ is the similarity degree between $\alpha_{\sigma(i)}$ and $\alpha$ is calculated by (8). $\alpha$ is the average value of $\alpha_{i}$ which is calculated by (10). And $\alpha_{\sigma(i)}$ is ith largest of $\alpha_{i}$ and with the condition satisfying $\alpha_{\sigma(i-1)} \geq \alpha_{\sigma(i)}$, $(i=1,2, \cdots, n)$. The weight calculations depend on the membership, nonmembership, and hesitation of IFNs. If the intuitionistic fuzzy value is closer to the average value, the weight value will be greater. If the intuitionistic fuzzy value is far away from the average value, the weight value will be smaller. It can represents the risk neutralness decisionmakers' attitude.

In order to aggregate risk attitude information, both the importance of IFNs and the risk factors brought by the hesitation of IFNs should be taken into consideration. We proposed an intuitionistic fuzzy dependent hybrid weighted operator (IFDHW), defined as follows.

Definition 7. $\alpha_{i}=\left(\mu_{i}, v_{i}\right)(i=1,2, \cdots, n)$ is a set of IFNs, and letting $\Theta$ be the set of intuitionistic fuzzy numbers, then IFDHW : $\Theta^{n} \longrightarrow \Theta$ is defined as follows:

$$
\begin{gathered}
\operatorname{IFDHW}_{\omega}\left(\alpha_{1}, \alpha_{2}, \cdots, \alpha_{n}\right)=\sum_{i=1}^{n} \omega_{i} \dot{\alpha}_{\sigma(i)} \\
=\left(1-\prod_{i=1}^{n}\left(1-\mu_{i}\right)^{\omega_{i}}, \prod_{i=1}^{n} v_{i}^{\omega_{i}}\right)
\end{gathered}
$$

then IFDHW is an intuitionistic fuzzy dependent hybrid weighted operator, where $\dot{\alpha}_{i}=n w_{i} \alpha_{i}, \dot{\alpha}_{\sigma(i-1)} \geq \dot{\alpha}_{\sigma(i)}$ for all $i$ and $W=\left(w_{1}, w_{2}, \cdots w_{n}\right)^{T}$ is the weight vector of $\alpha_{i}$ with the condition $\sum_{i=1}^{n} w_{i}=1(i=1,2, \cdots, n), w_{i} \in[0,1] . \mathrm{n}$ is called the balancing coefficient. $\omega_{i}$ is decided by decision-makers' three kinds of attitude: risk proneness, risk aversion, and risk neutralness. $\omega_{i}$ can be calculated by using (11)-(13). 


\section{Intuitionistic Fuzzy Multiple Attribute Decision-Making Method Based on Decision-Maker's Risk Attitude}

For solving a MADM problem with intuitionistic fuzzy information, let us suppose that $A=\left\{A_{1}, A_{2}, \cdots, A_{n}\right\}(i=$ $1,2, \cdots, \mathrm{n})$ is a set of $\mathrm{n}$ alternatives to be selected; $G=$ $\left\{G_{1}, G_{2}, \cdots, G_{m}\right\}(j=1,2, \cdots, m)$ is a set of $m$ attributes and whose weight vector is $W=\left(w_{1}, w_{2}, \cdots w_{m}\right)^{T}$, where $w_{j}$ is the weight for attribute $G_{j}$ with the condition $\sum_{i=1}^{n} w_{i}=1(i=$ $1,2, \cdots, n), w_{i} \in[0,1] . \mathrm{D}=\left(\alpha_{i j}\right)_{\mathrm{m} \times n}=\left(\left(\mu_{i j}, v_{i j}\right)\right)_{\mathrm{m} \times n}$ is the decision matrix, where $\alpha_{i j}$ is provided by decision-maker for alternative $A_{i}$ with respect to attribute $G_{j}$.

In the following three steps, we will use the IFDHW operator to solve MADM problems by developing a method based on decision-maker's risk attitude.

Step 1. The decision-maker gives the decision matrix $D=$ $\left(\alpha_{i j}\right)_{n \times m}$ according to the actual situation with weight vector $W=\left(w_{1}, w_{2}, \cdots w_{m}\right)^{T}$. Meanwhile, decision-maker chooses the appropriate risk weight equation to calculate $\omega_{i}$ according to the decision-maker's risk preference.

Step 2. Utilize the IFDHW operator and calculate overall values $r_{A_{i}}$ for all the alternatives $A_{i}(i=1,2, \cdots, n)$ by using (14).

Step 3. Based on aggregated value $r_{A_{i}}$ for all $A_{i}(i=$ $1,2, \cdots, n)$, the score values $S\left(\alpha_{i}\right)$ are calculated and ranked. The best one(s) of all the alternatives $A_{i}$ would be selected.

\section{Illustrated Example}

There is an investment company who want to invest in one of the robot manufacturing enterprises $A_{i}(i=1,2,3,4)$. The investment company has determined five attributes $G_{j}(j=1,2,3,4,5)$ to evaluate the robot manufacturing enterprises: production capacity; technological innovation ability; marketing ability; management ability; risk aversion ability. $w=(0.25,0.2,0.2,0.1,0.25)^{T}$ is the weight vector of these attributes. The intuitionistic fuzzy decision matrix is provided by the company which is listed in Table 1 . The investment decision-making steps are shown as follows.

Step 1. Using weight equation to calculate alternatives' risk weight vector, we take the one kind of decision-maker's attitude for an example. Using risk proneness weight equation $\omega_{i}=\left(1-\mu_{i}-v_{i}\right) / \sum_{i=1}^{n}\left(1-\mu_{i}-v_{i}\right)$ to calculate four robots manufacturing enterprises' risk weight vector

$$
\begin{aligned}
& \omega 1=(0.1667,0.3333,0.1667,0.1667,0.1667)^{T}, \\
& \omega 2=(0.1250,0.1250,0.1250,0.3750,0.2500)^{T}, \\
& \omega 3=(0.1000,0.3000,0.2000,0.3000,0.1000)^{T}, \\
& \omega 4=(00.1667,0.1667,0.000,0.1667,0.5000)^{T} .
\end{aligned}
$$

Step 2. Use the IFDHW operator. First, calculate $\dot{\alpha}_{i}=$ $n w_{i} \alpha_{i}$, taking alternative $\mathrm{A}_{1}$ for example. $\dot{\alpha}_{1}=(0.4054$, $0.503) \dot{\alpha}_{2}=(0.7000,0.1000), \dot{\alpha}_{3}=(0.7000,0.2000), \dot{\alpha}_{4}=$ $(0.3183,0.5946)$, and $\dot{\alpha}_{5}=(0.6464,0.2530)$. Then calculate overall values $r_{A_{i}}$ for every $A_{i}(i=1,2, \cdots, n)$ by using (14):

$$
\begin{aligned}
& r_{A_{1}}=(0.6038,0.2308), \\
& r_{A_{2}}=(0.5356,0.2753), \\
& r_{A_{3}}=(0.5146,0.2321), \\
& r_{A_{4}}=(0.5033,0.2669) .
\end{aligned}
$$

Step 3. Sort the alternatives by calculating the score functions $S\left(r_{A_{i}}\right),(i=1,2,3,4)$ for every alternatives based on overall values $r_{A_{1}}, r_{A_{2}}, r_{A_{3}}$, and $r_{A_{4}}$. If two or more score values are equal, then we can use accuracy function $H\left(r_{A_{i}}\right)$ to get the ranking results.

$$
\begin{aligned}
& S\left(r_{A_{1}}\right)=0.3729, \\
& S\left(r_{A_{2}}\right)=0.2603, \\
& S\left(r_{A_{3}}\right)=0.2825, \\
& S\left(r_{A_{4}}\right)=0.2364 .
\end{aligned}
$$

According to the results of $S\left(r_{A_{i}}\right),(i=1,2,3,4)$, and thus $\mathrm{A}_{1}>A_{3}>A_{2}>A_{4}$, where " $>$ " denotes "be superior to," therefore, for a decision-maker in the risk proneness attitude, $A_{1}$ is the best investment company. Using the same steps, the ranking results in other two cases are shown in Table 2.

Table 2 shows that rest on different risk attitudes the best alternatives can be different. For risk proneness attitude that the best investment company is $A_{1}$, for risk aversion attitude it is $A_{2}$ and for risk neutralness attitude it is $A_{3}$. This ranking method can reflect the impact of risk factors on the ranking results and can also choose the best alternative according to different risk attitudes of decision-makers.

\section{Conclusions}

In this paper, we want to solve the MADM problems when decision-makers take different risk attitude. The innovations of this paper are listed as follows:

(1) We introduced three risk preference attitudes of decision-makers to MADM field. Three risk preference attitudes are from risk preference partition theory which is contained in expected utility theory.

(2) Considering the hesitation information of IFNs, we defined three equations for constructing weight vectors according to different decision-makers' attitudes. The weight vectors are subjective and easy to calculate for solving the MADM problems with lots of alternatives and attributes.

(3) This decision-making method can provide decisionmaking basis for many different fields when decision-makers want to check if there are any differences while they are 
TABLE 1: Intuitionistic fuzzy decision matrix for investment.

\begin{tabular}{lccccc}
\hline & $\mathrm{G}_{1}$ & $\mathrm{G}_{2}$ & $\mathrm{G}_{3}$ & $\mathrm{G}_{4}$ & $\mathrm{G}_{5}$ \\
\hline$A_{1}$ & $(0.5,0.4)$ & $(0.7,0.1)$ & $(0.7,0.2)$ & $(0.4,0.5)$ & $(0.5,0.4)$ \\
$A_{2}$ & $(0.6,0.3)$ & $(0.5,0.4)$ & $(0.7,0.2)$ & $(0.3,0.4)$ & $(0.6,0.2)$ \\
$A_{3}$ & $(0.7,0.2)$ & $(0.6,0.1)$ & $(0.5,0.3)$ & $(0.4,0.3)$ & $(0.5,0.4)$ \\
$A_{4}$ & $(0.5,0.4)$ & $(0.5,0.4)$ & $(0.6,0.4)$ & $(0.6,0.3)$ & $(0.4,0.3)$ \\
\hline
\end{tabular}

TABLE 2: Ranking results of different risk attitudes.

\begin{tabular}{lc}
\hline Risk attitudes & Ranking results \\
\hline Risk proneness & $\mathrm{A}_{1}>A_{3}>A_{2}>A_{4}$ \\
Risk aversion & $\mathrm{A}_{2}>A_{3}>A_{1}>A_{4}$ \\
Risk neutralness & $\mathrm{A}_{3}>A_{2}>A_{1}>A_{4}$ \\
\hline
\end{tabular}

in different attitudes. Then they can get the most desirable alternative(s).

In the future, we should study the accuracy of this proposed method. Meanwhile, we can also extend the proposed method to solve the MAGDM problems.

\section{Data Availability}

The intuitionistic fuzzy data used to support the findings of this study are included within the article.

\section{Conflicts of Interest}

The authors declare that there are no conflicts of interest regarding the publication of this paper.

\section{Acknowledgments}

This paper is supported by the Key Research Institute of Philosophy and Social Science of Zhejiang Province (Modern Port Service Industry and Creative Culture Research Center) (nos. 16JDGH067, 15JDLG01YB), Research Project of Philosophy and Social Science of Zhejiang Province (no. 18NDJC283YB), and Soft Science Project of Ningbo (nos. 2017A10085, 2017A10068)

\section{References}

[1] L. A. Zadeh, "Fuzzy sets," Information and Control, vol. 8, pp. 338-353, 1965.

[2] K. T. Atanassov, "Intuitionistic fuzzy sets," Fuzzy Sets and Systems, vol. 20, no. 1, pp. 87-96, 1986.

[3] Z. S. Xu and R. R. Yager, "Some geometric aggregation operators based on intuitionistic fuzzy sets," International Journal of General Systems, vol. 35, no. 4, pp. 417-433, 2006.

[4] Z. Xu, "Intuitionistic fuzzy aggregation operators," IEEE Transactions on Fuzzy Systems, vol. 15, no. 6, pp. 1179-1187, 2007.

[5] S. Zeng, "Pythagorean fuzzy multiattribute group decision making with probabilistic information and OWA approach," International Journal of Intelligent Systems, vol. 32, no. 11, pp. 1136-1150, 2017.
[6] G. Wei, "Some induced geometric aggregation operators with intuitionistic fuzzy information and their application to group decision making," Applied Soft Computing, vol. 10, no. 2, pp. 423-431, 2010.

[7] Z. Su, G. Xia, M. Chen, and L. Wang, "Induced generalized intuitionistic fuzzy OWA operator for multi-attribute group decision making," Expert Systems with Applications, vol. 39, no. 2, pp. 1902-1910, 2012.

[8] S. Zeng, Z. Mu, and T. Baležentis, "A novel aggregation method for Pythagorean fuzzy multiple attribute group decision making," International Journal of Intelligent Systems, vol. 33, no. 3, pp. 573-585, 2018.

[9] C. Q. Tan, "A multi-criteria interval-valued intuitionistic fuzzy group decision making with Choquet integral-based TOPSIS," Expert Systems with Applications, vol. 38, no. 4, pp. 3023-3033, 2011.

[10] F. E. Boran, S. Genç, M. Kurt, and D. Akay, "A multi-criteria intuitionistic fuzzy group decision making for supplier selection with TOPSIS method," Expert Systems with Applications, vol. 36, no. 8, pp. 11363-11368, 2009.

[11] S. Zeng and Y. Xiao, "A method based on topsis and distance measures for hesitant fuzzy multiple attribute decision making," Technological and Economic Development of Economy, vol. 24, no. 3, pp. 969-983, 2018.

[12] K. Chatterjee, M. B. Kar, and S. Kar, "Strategic Decisions Using Intuitionistic Fuzzy Vikor Method for Information System (IS) Outsourcing," in Proceedings of the International Symposium on Computational and Business Intelligence IEEE Computer Society, pp. 123-126, 2013.

[13] H. Liao and Z. Xu, "A VIKOR-based method for hesitant fuzzy multi-criteria decision making," Fuzzy Optimization and Decision Making, vol. 12, no. 4, pp. 373-392, 2013.

[14] K. Chatterjee and S. Kar, "Unified Granular-number-based AHP-VIKOR multi-criteria decision framework," Granular Computing, vol. 2, no. 3, pp. 199-221, 2017.

[15] Y. Huang, G. Wei, and C. Wei, "VIKOR Method for Interval Neutrosophic Multiple Attribute Group Decision-Making," Information, vol. 8, no. 4, p. 144, 2017.

[16] F. Meng, C. Tan, and X. Chen, “An approach to Atanassov's interval-valued intuitionistic fuzzy multi-attribute decision making based on prospect theory," International Journal of Computational Intelligence Systems, vol. 8, no. 3, pp. 591-605, 2015.

[17] J. D. Qin, X. W. Liu, and W. Pedrycz, "An extended VIKOR method based on prospect theory for multiple attribute decision making under interval type-2 fuzzy environment," KnowledgeBased Systems, vol. 86, pp. 116-130, 2015.

[18] N. Xie, Z. Li, and G. Zhang, "An intuitionistic fuzzy soft set method for stochastic decision-making applying prospect theory and grey relational analysis," Journal of Intelligent \& Fuzzy Systems: Applications in Engineering and Technology, vol. 33, no. 1, pp. 15-25, 2017. 
[19] Peng Li, Yingjie Yang, and Cuiping Wei, "An Intuitionistic Fuzzy Stochastic Decision-Making Method Based on CaseBased Reasoning and Prospect Theory," Mathematical Problems in Engineering, vol. 2017, Article ID 2874954, 13 pages, 2017.

[20] Z. S. Xu, "An overview of methods for determining OWA weights," International Journal of Intelligent Systems, vol. 20, no. 8, pp. 843-865, 2005.

[21] X. Guo, Z. Yuan, and B. Tian, "Supplier selection based on hierarchical potential support vector machine," Expert Systems with Applications, vol. 36, no. 3, pp. 6978-6985, 2009.

[22] A. Zouggari and L. Benyoucef, "Simulation based fuzzy TOPSIS approach for group multi-criteria supplier selection problem," Engineering Applications of Artificial Intelligence, vol. 25, no. 3, pp. 507-519, 2012.

[23] G. Wang, S. H. Huang, and J. P. Dismukes, "Product-driven supply chain selection using integrated multi-criteria decisionmaking methodology," International Journal of Production Economics, vol. 91, no. 1, pp. 1-15, 2004.

[24] J. Liu, S.-F. Liu, P. Liu, X.-Z. Zhou, and B. Zhao, "A new decision support model in multi-criteria decision making with intuitionistic fuzzy sets based on risk preferences and criteria reduction," Journal of the Operational Research Society, vol. 64, no. 8, pp. 1205-1220, 2013.

[25] S. Wan, F. Wang, and J. Dong, "A Three-Phase Method for Group Decision Making with Interval-Valued Intuitionistic Fuzzy Preference Relations," IEEE Transactions on Fuzzy Systems, vol. 26, no. 2, pp. 998-1010, 2018.

[26] Y. Lin and Y. Wang, "Group decision making with consistency of intuitionistic fuzzy preference relations under uncertainty," IEEE/CAA Journal of Automatica Sinica, vol. 5, no. 3, pp. 741748, 2018.

[27] R. Roostaee, M. Izadikhah, F. H. Lotfi, and M. RostamyMalkhalifeh, "A multi-criteria intuitionistic fuzzy group decision making method for supplier selection with vikor method," International Journal of Fuzzy System Applications, vol. 2, no. 1, pp. 1-17, 2012.

[28] G. Büyüközkan and F. Göçer, "Application of a new combined intuitionistic fuzzy MCDM approach based on axiomatic design methodology for the supplier selection problem," Applied Soft Computing, vol. 52, pp. 1222-1238, 2017.

[29] W. L. Hung and M. S. Yang, "On the J -divergence of intuitionistic fuzzy sets with its application to pattern recognition," Information Sciences, vol. 178, no. 6, pp. 1641-1650, 2008.

[30] S.-H. Cheng, S.-M. Chen, and T.-C. Lan, "A New Similarity Measure between Intuitionistic Fuzzy Sets for Pattern Recognition Based on the Centroid Points of Transformed Fuzzy Numbers," in Proceedings of the IEEE International Conference on Systems, Man, and Cybernetics IEEE, pp. 1125-1129, 2015.

[31] H. Nguyen, "A novel similarity/dissimilarity measure for intuitionistic fuzzy sets and its application in pattern recognition," Expert Systems with Applications, vol. 45, pp. 97-107, 2016.

[32] Z. S. Xu and R. R. Yager, "Intuitionistic and interval-valued intutionistic fuzzy preference relations and their measures of similarity for the evaluation of agreement within a group," Fuzzy Optimization and Decision Making, vol. 8, no. 2, pp. 123-139, 2009. 


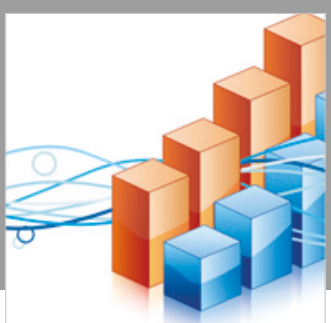

Advances in

Operations Research

\section{-n-m}
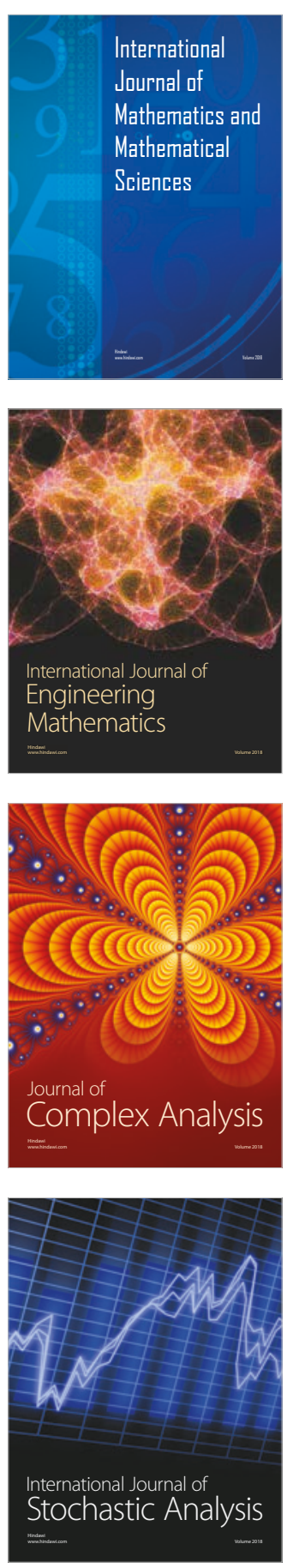
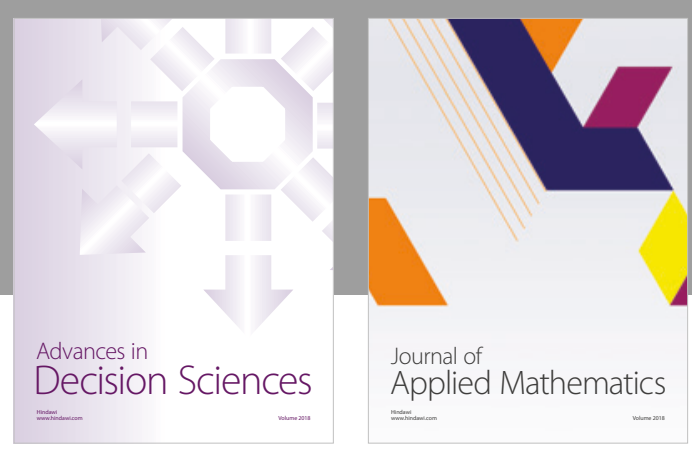

Journal of

Applied Mathematics
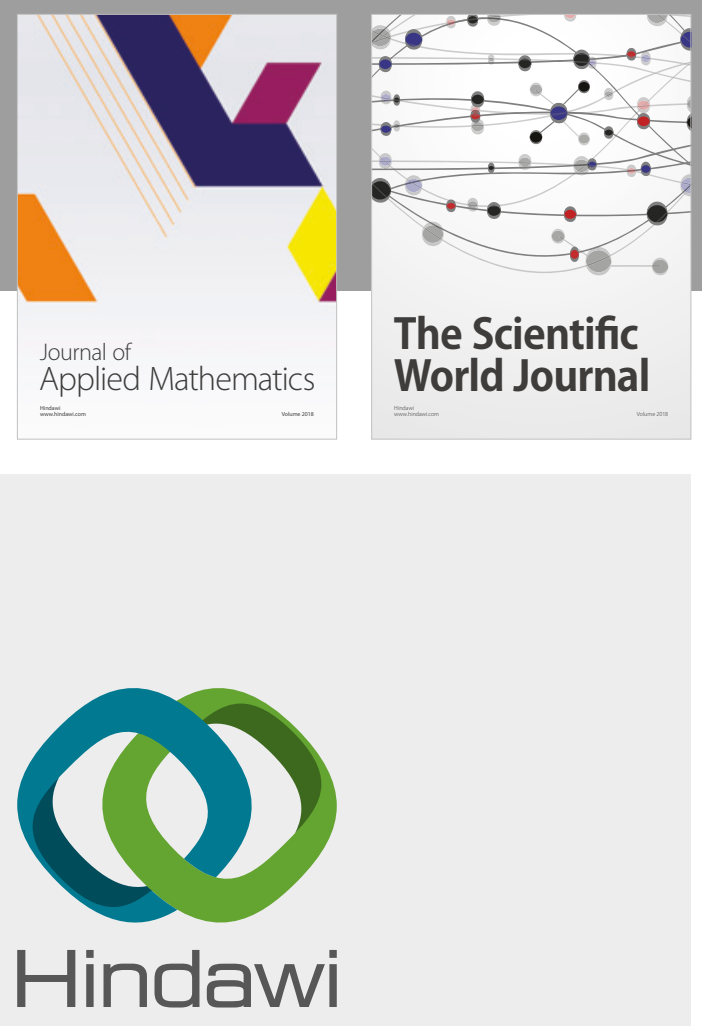

Submit your manuscripts at

www.hindawi.com

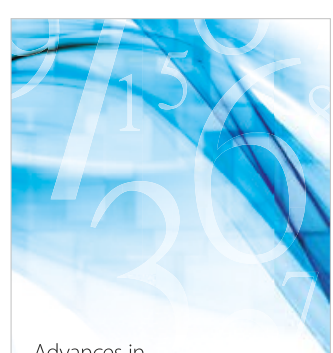

Advances in
Numerical Analysis
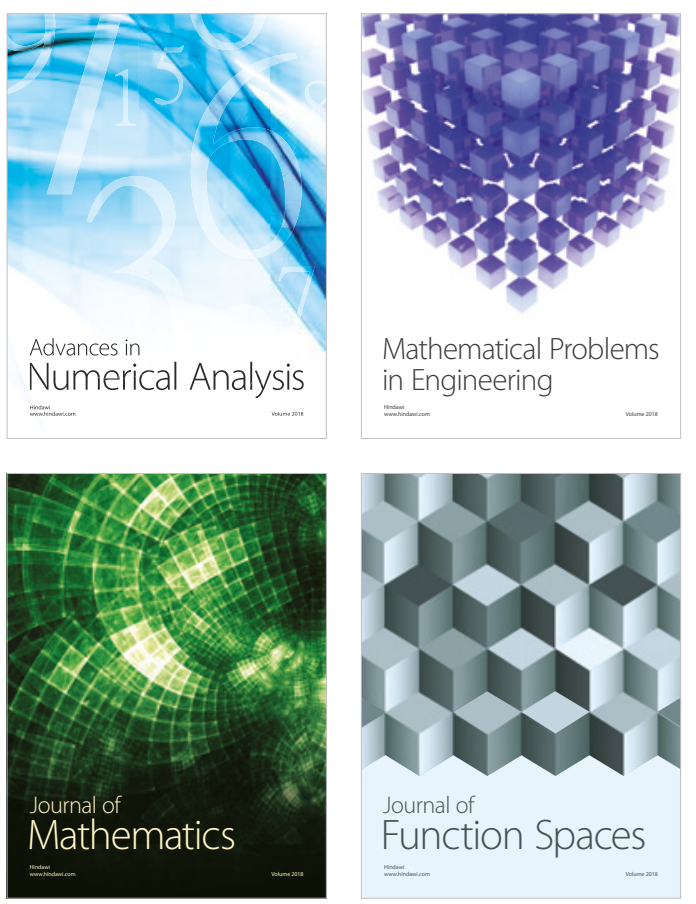

Mathematical Problems in Engineering

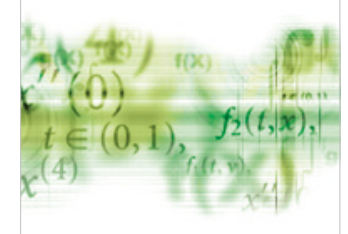

International Journal of

Differential Equations

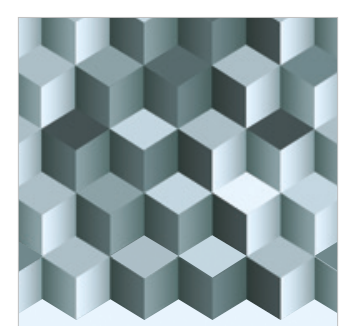

Journal of

Function Spaces
The Scientific

World Journal

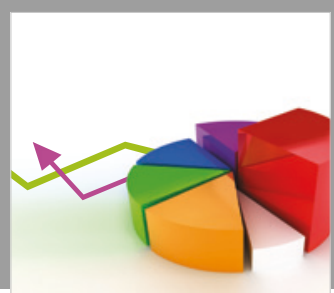

Journal of

Probability and Statistics
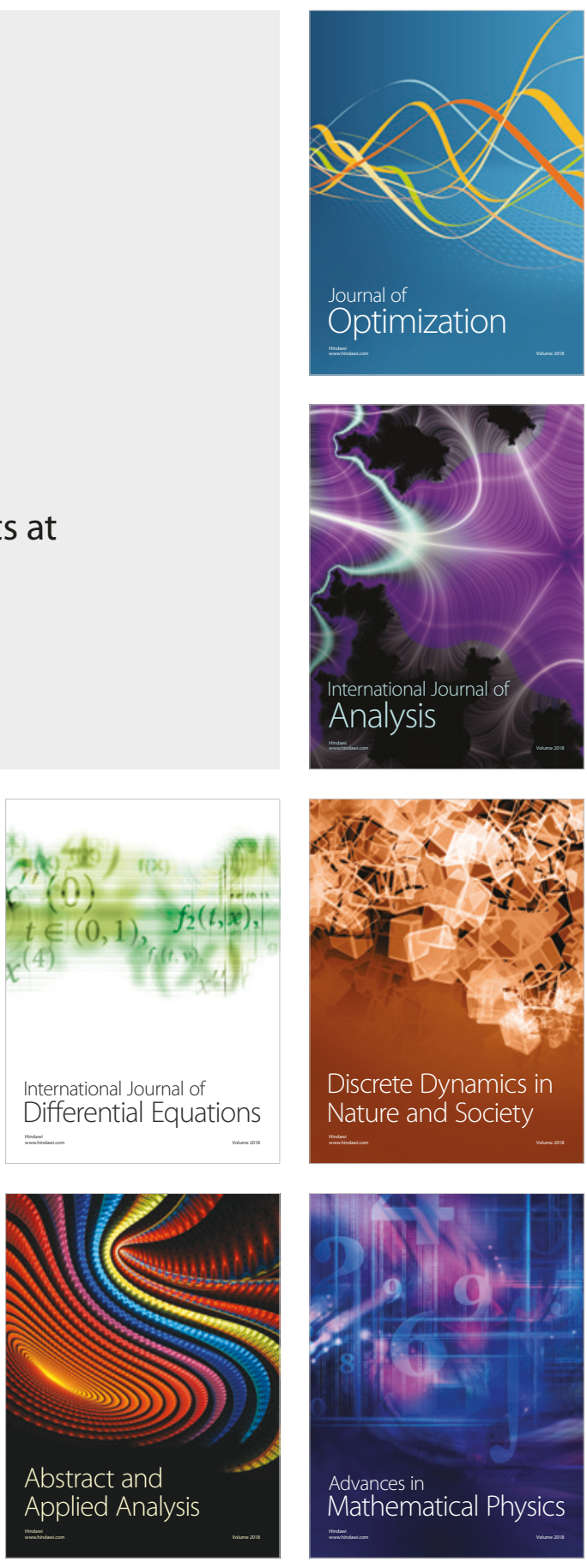